\title{
Confluences in Programming Languages Research (Keynote)
}

\author{
David Walker \\ Princeton University, USA \\ dpw@cs. princeton.edu
}

\begin{abstract}
A confluence occurs when two rivers flow together; downstream the combined forces gather strength and propel their waters forward with increased vigor. In academic research, Varghese argues a confluence occurs after some trigger-perhaps a discovery or a change in technology - and brings two previously separate branches of research together [1]. In this talk, I will discuss confluences in programming languages research. Here, confluences often occur when basic research finds application in some important new domain. Two prime examples from my own career involve the confluence of research in type theory and systems security, triggered by new theoretical tools for reasoning about programming language safety, and the confluence of formal methods and networking, triggered by the rise of data centers. These experiences may shed light on what to teach our students and what is next for programming languages research.
\end{abstract}

Categories and Subject Descriptors D.1 [Programming Techniques]

Keywords programming languages, basic research, interdisciplinary research, formal methods, network programming

\section{Acknowledgements}

This work is supported in part by the NSF under grant CNS1111520 and a gift from Cisco. Any opinions, findings, and conclusions or recommendations expressed in this material are those of the author and do not necessarily reflect the views of the National Science Foundation or Cisco.

\section{Biography}

David Walker is a Professor of Computer Science at Princeton University, having received tenure in July 2008 and the rank of full professor in July 2013. He received his B.Sc. from Queen's University, Canada in 1995 and his Ph.D. in Computer Science from Cornell University in 2001. His research interests include programming language theory, design and implementation of all kinds. He is especially interested in type systems and the development of new domain-specific programming languages.

After arriving at Princeton in February 2002, he won an NSF Career award in 2003 and an Alfred Sloan Fellowship in 2004. In 2007, with his students and colleagues at Princeton, he won the PLDI best paper award for the paper entitled Fault-Tolerant Typed Assembly Language. In 2008, his paper From System F to Typed Assembly Language, co-authored with Greg Morrisett, Karl Crary and Neal Glew, won a 10-year retrospective award for most influential POPL 1998 paper. In 2013, with his students and colleagues at Princeton and Cornell, he won the NSDI community award for his paper on Composing Software-Defined Networks. He served as an associate editor for ACM Transactions on Programming Languages and Systems from 2007-2015 and as the Program Chair for the 42nd ACM Symposium on Principles of Programming Languages, POPL 2015. He won the ACM SIGPLAN Robin Milner Young Researcher Award in 2015.

\section{References}

[1] Life in the Fast Lane: Viewed from the Confluence Lens. George Varghese. In ACM SIGCOMM Computer Communication Review, Volume 45, Issue 1, January 2015.
Permission to make digital or hard copies of part or all of this work for personal or classroom use is granted without fee provided that copies are not made or distributed for profit or commercial advantage and that copies bear this notice and the full citation on the first page. Copyrights for third-party components of this work must be honored. For all other uses, contact the Owner/Author.

Copyright is held by the owner/author(s).

POPL'16, January 20-22, 2016, St. Petersburg, FL, USA

ACM. 978-1-4503-3549-2/16/01 ..\$15.00

http://dx.doi.org/10.1145/2837614.2843896 